\title{
Neutrosophic Function for Assessing the Scale Effect of the Rock Joint Surface Roughness
}

\author{
Rui Yong $\mathbb{D}^{1,2}$ Shaonan Tan $(\mathbb{D})^{1}$ Jun Ye $\mathbb{D}^{1,2}$ and Shigui Du $\mathbb{D}^{1,2}$ \\ ${ }^{1}$ Key Laboratory of Rock Mechanics and Geohazards of Zhejiang Province, Shaoxing University, Shaoxing, \\ Zhejiang 312000, China \\ ${ }^{2}$ School of Civil and Environmental Engineering, Ningbo University, Ningbo, Zhejiang 315211, China
}

Correspondence should be addressed to Rui Yong; yongrui_usx@hotmail.com

Received 19 December 2020; Revised 1 June 2021; Accepted 23 June 2021; Published 6 July 2021

Academic Editor: Mijia Yang

Copyright (C) 2021 Rui Yong et al. This is an open access article distributed under the Creative Commons Attribution License, which permits unrestricted use, distribution, and reproduction in any medium, provided the original work is properly cited.

\begin{abstract}
A new investigation method is proposed for recording large-sized joint profiles and making statistical analyses of the joint roughness coefficient (JRC) values of the $10-300 \mathrm{~cm}$ sized profiles. The mechanical hand profilograph is used for joint roughness measurement due to its advantage of easy operation and high accuracy in recording joint traces. Based on the proposed method, it provides sufficient samples from various positions on the large joint profile, which allows the statistical evaluation of JRC values. A neutrosophic number (NN) is employed for revealing determinate and/or indeterminate information as it consists of determinate and indeterminate parts. Due to the uncertainty of JRC in the real world, NN is chosen to represent the JRC value, which is not only random but also a fuzzy indefinite parameter. The neutrosophic function is used to analyze and express the scale effect of joint surface roughness, and its derivative is used to describe the changing trend of the scale effect. The results show that the JRC value of the joint profile is related to the scale and has a negative effect on the surface roughness of the rock joint. The indeterminate information about the scale effect on joint roughness is described by the neutrosophic functions, and the derivative indicated that the JRC values of small samples are more sensitive than those of large-sized examples. When the length of the sample exceeds the stationarity limit of $80 \mathrm{~cm}$, the roughness appears to be almost scale independent.
\end{abstract}

\section{Introduction}

Rock joint roughness plays an important role in hydraulic and mechanical characteristics of discontinuous rock masses [1-4]. In the past few decades, considerable efforts have been devoted to the investigation of the basic properties of joint roughness. In practice, it is found that roughness is usually scale dependent, and the scale effect on roughness is very important for engineering design [5]. This delivers an urgent need to more accurately determine the relationship between roughness and sample size.

To study the scale effect, the roughness of samples of different sizes should be measured from the same joint surface. Therefore, the method used to measure the roughness should provide a quick and effective measurement of the joint profile in length ranging from a few centimeters to meters. Generally, the joint roughness measurement methods can be classified into "contact methods" and "noncontact methods" [4]. Due to their advantages of easy operation and low cost, the contact method is usually used to measure the topography of rock discontinuities. Weissbach [6] noted that the most reliable method of characterizing a surface in three dimensions remains a combination of a set of linear profile measurements using a stylus instrument. Mogilevskaya [7] measured the macroscopic relief of a joint surface with a VNIIG needle-tip meter. Micallef and Williams [8] used an $\mathrm{H}$-shaped rock profiler to measure locally common smooth and irregular natural rock surfaces. Alameda-Hernández et al. [9] applied a needle profilometer and image-processing method for fast and economical digitization of rock surface profiles. Although these methods can obtain high-resolution and high-precision joint profiles, the allowable size of the measured joint is usually very limited $(\leq 1 \mathrm{~m})$, because the digitization of a 
large joint is very time-consuming and inconvenient to operate in situ. Recently, the noncontacted instruments have emerged as an alternative for quickly and accurately measuring joint surfaces in both laboratory and situ environments [2]. To investigate the scale-dependency of surface roughness, Fardin et al. [10] digitized a natural rock joint surface replica in the laboratory using a laser scanner with high accuracy and resolution. Feng et al. [11] employed a total station for making high in situ noncontact measurements of fracture roughness. This method was proved to have a similar accuracy level as that of laser scanners. Haneberg [12] introduced a method to obtain outcrop-scale directional roughness profiles based on three-dimensional photogrammetric or laser scanner point clouds. Tatone and Grasselli [2] used a laser scanning system to digitize the surface roughness of large-scale rock fractures in situ. The noncontact methods greatly improved the speed and accuracy of roughness measurements. However, the accuracy and precision of measurement are affected by operation conditions such as surface covering, light requirement, and color distortions caused by rock components [13]. In addition, the measurement noise involved in the data is another fundamental limitation [14].

The surface roughness of rock joints varies with the sample scale and location. The joint surface roughness varies significantly with position, even when measured on the same joint and along the same direction [15]. Ye et al. [16] and Liu et al. [17] studied the roughness of natural joint profiles, and the results showed that the surface roughness differs with positions of the large joint. Roughness heterogeneity affects the results of scale-dependent investigations, especially when joint samples are insufficient. Barton [18] concluded that sampling bias and sampling disturbance influenced the reliability of the previous findings of scale effect on joint roughness. Some researchers have found that statistical analysis of roughness helps reveal the scale effect more accurately. Du et al. [19] analyzed the relationship between the asperity amplitudes of joint profiles and sample size, and a negative scale effect was observed. However, Hsiung et al. [20] stated that the average value of JRC statistical data is difficult to represent the global roughness of a given joint due to its variation. Chen et al. [21] and Wang et al. [22] applied the geological statistics method to investigate the anisotropy and the scale effect of joint roughness. Li et al. [23] studied the fractal dimensions of waviness and unevenness in sampling window sizes ranging from $100 \mathrm{~mm} \times 100 \mathrm{~mm}$ to $1000 \mathrm{~mm} \times 1000 \mathrm{~mm}$ and found that there is no obvious stationarity threshold for rock joint samples. Yan et al. [24] found that the joint roughness scale effect depends on shear directions, and it tends to be stable when the scale of the study increases to a certain value. Due to the irregular and inconsistent properties of the joint surface, it is still difficult to establish a function for quantitatively expressing the statistical results of roughness to accommodate different sample sizes.

To address these issues, this study represents a new investigation method to analyze the scale dependency of rock joint surface roughness. The rest of the paper is organized as follows. Section 2 contains the literature review on some limitations in previous studies on scale effect. Section 3 contains the measurement method and description of the scale effect of joint surface by neutrosophic functions. Section 4 contains the application of the proposed method in a case study. Section 5 contains the conclusions.

\section{Limitations in Previous Studies on Scale Effect}

First, the lack of appropriate devices hinders accurate and quick measurement of field-scale joint surfaces. It is widely recognized that joint roughness consists of a large-scale waviness component and a small-scale unevenness component [25]. Investigations of rock joint surface roughness were carried out based on small samples in previous studies. Recently, the optical instrument has been proved to be a good option for measuring large joint surfaces in situ $[13,26,27]$. Yet there are disadvantages, such as the cost of the equipment, relevant software, and costs of training. In rock engineering practices, conventional contact profiling methods are still used in joint roughness measurements. These inexpensive methods can provide repeatable measures of joint traces that are ready to be digitized and analyzed. The simple profile gauge and $10 \mathrm{~cm}$ long steel stylus comb are habitually used in the field for estimating the small-scale joint roughness [28]. But these devices/apparatus (e.g., profile comb, electrical profilograph, and needle profilometer) cannot measure the joint profiles with several meters or even tens of meters, in length. As a result, largescale roughness components are rarely measured and ignore the mechanical impact of field-scale discontinuities [10].

Second, the sampling bias and disturbance affect the reliability of the previous findings concerning some of the apparent scale effects [18]. Numerous potential scale effects are evident in rock mechanics. Unfortunately, conflicting results about scale effects on rock joint surface roughness were produced in previous studies. Indeed, a lot of confusion was caused by the difficulties in obtaining representative samples. Joint surface roughness varies not only from joint to joint but also with scale and the measurement locations [29].

The following example was used to show the JRC values over a $100 \mathrm{~cm}$ long specimen. Here, the JRC values of different sized profiles were calculated based on the method proposed by Zhang et al. [30]. The detailed roughness determination process is introduced as follows.

First, assume that a horizontal reference line crosses the selected profile. The average vertical distance between this horizontal reference line and the points on the digitized profile is obtained by

$$
h_{a v}=\frac{\int_{x=0}^{x=L}|y| \mathrm{d} x}{L}=\sum_{i=1}^{N} \frac{\left|y_{i+1}+y_{i}\right|\left(x_{i+1}-x_{i}\right)}{2 L}
$$

where $L$ is the digitized profile length and $x$ and $y$ are the $x$ and $y$-coordinates of the points on the profile.

Then, the modified root mean square $Z_{2}^{\prime}$ is determined as follows: 


$$
Z_{2}^{\prime}=\sqrt{\frac{1}{L} \int_{x=0}^{x=L}\left(\max \left(0, \frac{\mathrm{d} y}{\mathrm{~d} x}\right)\right)^{2} \mathrm{~d} x}=\left[\sum_{i=1}^{M} \frac{\left(\max \left(0, y_{i+1}-y_{i}\right)\right)^{2}}{\left(x_{i+1}-x_{i}\right) L}\right]^{1 / 2}
$$

Based on the logistic correlation, the JRC value can be determined as

$$
\mathrm{JRC}=\frac{40}{1+e^{-20 \lambda}}-20
$$

with

$$
\lambda=\left(\frac{h}{L}\right)^{\alpha} \cdot\left(Z_{2}^{\prime}\right)^{1-\alpha}, \quad \alpha=\frac{1}{3},
$$

where $\lambda$ is the roughness index.

This procedure was repeated to determine the JRC evaluation for all profiles.

In Figure 1, a digitized profile was separated using different sampling lengths $(10 \mathrm{~cm}, 20 \mathrm{~cm}$, and $50 \mathrm{~cm})$. Their JRC values are plotted in Figure 2. The JRC values changed with sample length and showed a negative scale effect. In addition, the JRC values change because of the positions of the joint samples. For example, the JRC values of the profiles in length of $10 \mathrm{~cm}$ ranged from 7.80 to 20 and from 5.26 to 12.90 for the $20 \mathrm{~cm}$ long joint samples. The JRC values of smaller samples showed a higher disparity/variation. Although the JRC mean values of small-sized samples were higher than the larger ones, some individual small samples showed lower roughness than the larger samples. Furthermore, as the surface roughness of joint samples between the adjacent sampling lengths was neglected, the characteristics of joint roughness cannot be considered comprehensively. For example, there were two $50 \mathrm{~cm}$ long samples in the range of $0-50 \mathrm{~cm}$ and $50-100 \mathrm{~cm}$ in Figure 1, but the roughness of the joint profile was in the range of $25-75 \mathrm{~cm}$. Based on these two samples, it was impossible to obtain a JRC value that could truly reflect the integrated characteristic of the $50 \mathrm{~cm}$ long joints. Also, only one joint sample could be acquired when the sample size was larger than $50 \mathrm{~cm}$. Thus, researchers may arbitrarily select the sampling regions from a large joint surface. Moreover, inadequate small-sized samples cannot comprehensively represent the real characteristic of joint roughness. It is therefore of urgent need to have generally adopted methods for obtaining representative samples.

\section{A New Survey Method}

3.1. Profile Recording Equipment. The profiles of rock joints were measured using mechanical hand profilograph (Figure 3). The profilograph is composed of several parts, including feeler, drawing pen, balance block, fixed board, bubble levels, and drawing paper. The top of the feeler, which is $80 \mathrm{~mm}$ long, is linked to the end of the balance block via a spring. The bottom consists of a steel needle. A small steel ball is mounted on the feeler point that keeps in contact with the rock joint surface during the measuring process. The drawing pen is fixed on the balance block and synchronized with it, moving up and down depending on the geometry of the joint surface. The fixed board is used to adjust and underlay the drawing paper. The board and paper must be kept perpendicular to the joint surface during measurement to avoid the "sudden jump" plotting problem when imitating the rock joint profile. Meanwhile, the balance block-feelerdrawing pen should also be perpendicular to the fixed board $\left(l_{1} \perp l_{2}, l_{2} \perp l_{3}, l_{1} \perp l_{4}, l_{1} \perp l_{3}\right.$, and $l_{2} / / l_{4}$ in Figure 3).

To measure the profiles in lengths of $200 \mathrm{~cm}, 300 \mathrm{~cm}$, $400 \mathrm{~cm}, 500 \mathrm{~cm}, 600 \mathrm{~cm}, 700 \mathrm{~cm}, 800 \mathrm{~cm}, 900 \mathrm{~cm}$, and $1000 \mathrm{~cm}$, stainless steel boards made of aluminum alloy were attached. This board, which was $2 \mathrm{~mm}$ thick, $100 \mathrm{~cm}$, or $50 \mathrm{~cm}$ in length and $20 \mathrm{~cm}$ in width (Figure $4(\mathrm{a})$ and Figure $4(\mathrm{~b})$ ), held the underlying drawing paper in place, providing stiffness and lightweight. Two lines of mounting holes, $5 \mathrm{~mm}$ in diameter, were set along the length of the board at an interval of $10 \mathrm{~cm}$, beginning $5 \mathrm{~cm}$ from the left end. The distance from the center of the mounting hole to the near margin was $2 \mathrm{~cm}$. The front and back layers are mutually staggered and connected to each other with bolts crossing the mounting holes. For example, a $200 \mathrm{~cm}$ long fixed board was assembled as shown in Figures 4(c) and 4(d). The fixed board was then carefully laid on the unfolded drawing paper (Figure 4(e)). The drawing paper was tightly rolled around the fixed board and held place with clamps. This is vital as the paper for recording joint profiles should be completely flat without any wrinkles. By performing this process, the profiles of large-scale rock joints can be copied accurately on the drawing paper. These profiles were then digitized using the grayscale image processing technique. More details of the profilograph can be found in [3]. Finally, the joint roughness was determined according to the digitized profile data. The sampling resolution has a significant impact on the joint roughness measurement [4]. In this study, the joint profiles were digitized with a sampling interval (SI) of $0.5 \mathrm{~mm}$, which is a very common sampling resolution used in previous studies on joint roughness determination.

3.2. Profile Sampling Process. Figures 2 and 3 show that the joint roughness of the profiles differs depending on the sampling locations. These samples, obtained by equally dividing the original joint profile, were inadequate yet. Consequently, they cannot accurately represent the real roughness characteristics. The sampling procedure is shown in Figure 5. First, it is assumed that the number of joint profiles in length of $l$ is $n$. The overlap length is $\lambda$ and the remaining length is $k$ :

$$
\begin{aligned}
& \lambda=\frac{l \cdot n-L}{n-1}, \\
& k=\frac{L-l}{n-1} .
\end{aligned}
$$

A large joint profile $(L=800 \mathrm{~cm}$ ) was considered to show how to extract the small-sized samples $(l=200 \mathrm{~cm})$. Seven samples $(n=7)$ are required to be obtained from the original joint profile. The overlap of two adjacent samples and the 


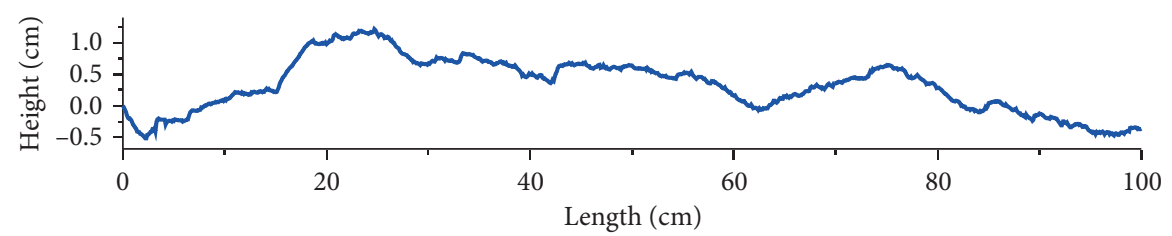

(a)

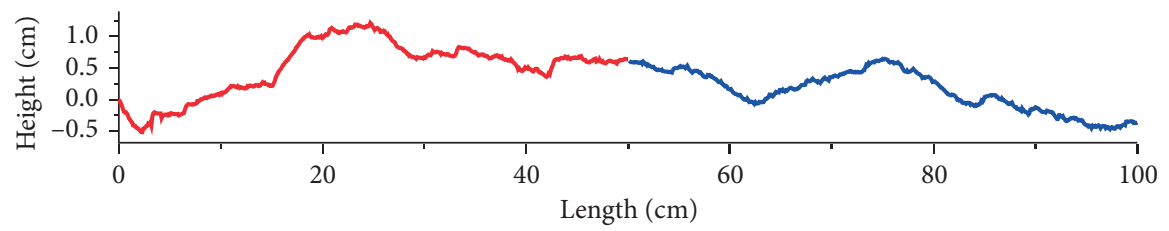

(b)

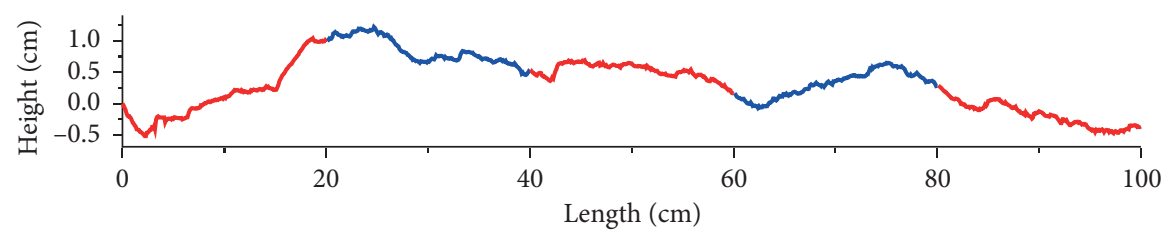

(c)

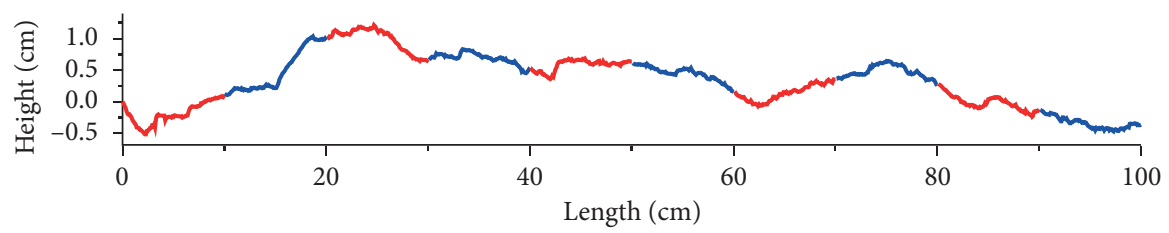

(d)

FIgURE 1: The digitized $100 \mathrm{~cm}$ long joint profile and the segmental samples obtained from the sampling lengths of $10 \mathrm{~cm}, 20 \mathrm{~cm}$, and $50 \mathrm{~cm}$. (a) $100 \mathrm{~cm}$ long joint sample. (b) $50 \mathrm{~cm}$ long joint sample. (c) $20 \mathrm{~cm}$ long joint sample. (d) $10 \mathrm{~cm}$ long joint sample.

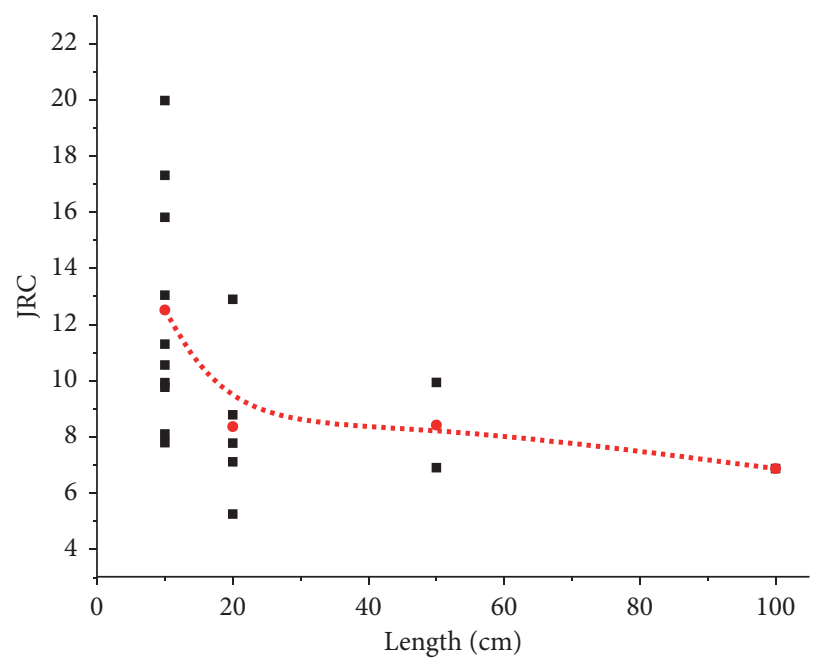

FIgURE 2: The JRC values of different sized joint profiles over the whole specimen.

length of the remaining part were calculated using (5) and (6), where the overlapped length $\lambda=100 \mathrm{~cm}$ and the length of the remaining part $k=100 \mathrm{~cm}$. Although it is unlike the independent samples obtained by equally dividing the original joint profile, it exposited more refined and integrated roughness characteristics of the rock joint. Samples from different positions of the rock joint were obtained as far as possible. Each sampling occurrence is independent, and thus, the statistical regularities of different sized samples could be analyzed. 


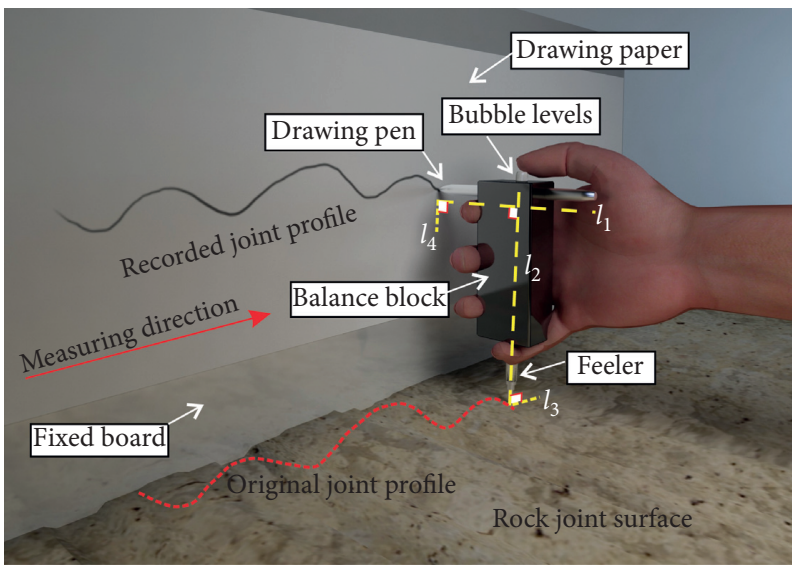

Figure 3: Mechanical hand profilograph [31].

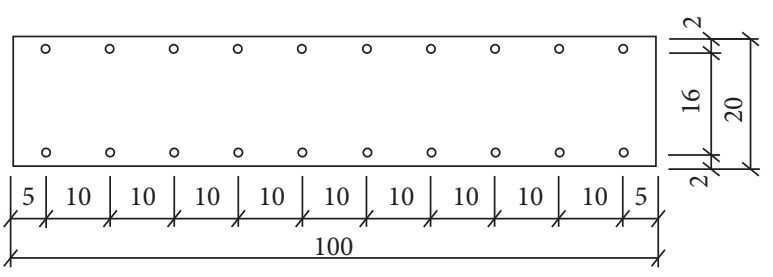

(a)

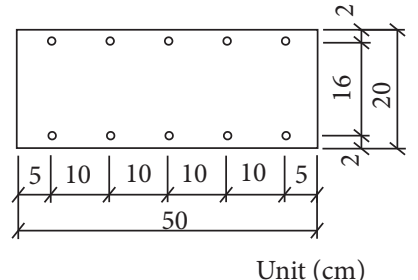

(b)

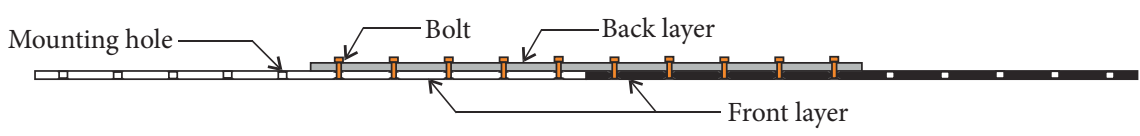

(c)

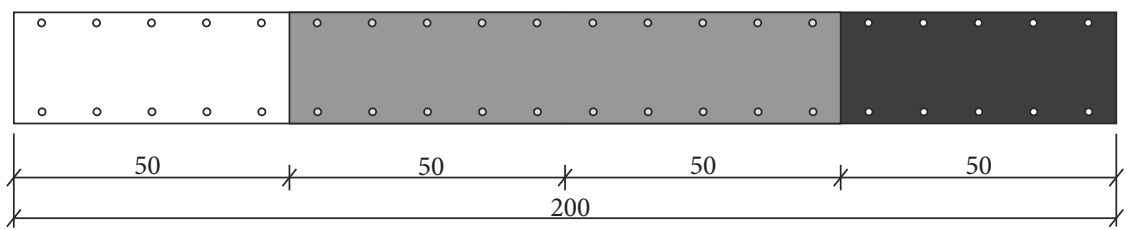

Unit $(\mathrm{cm})$

(d)

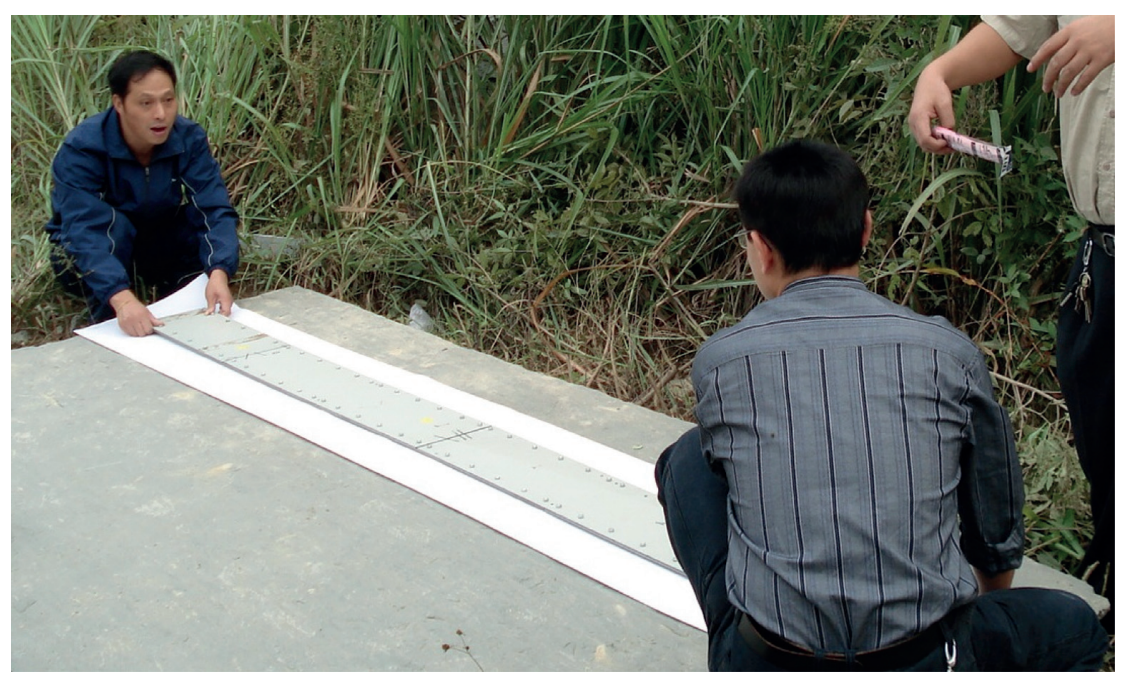

(e)

Figure 4: Fixed board and its assembling. (a) The geometry of $100 \mathrm{~cm}$ long basic board; (b) the geometry of $50 \mathrm{~cm}$ long basic board; (c) the side view of $200 \mathrm{~cm}$ long fixed board; (d) the top view of $200 \mathrm{~cm}$ long fixed board; (e) the installation of drawing paper. 


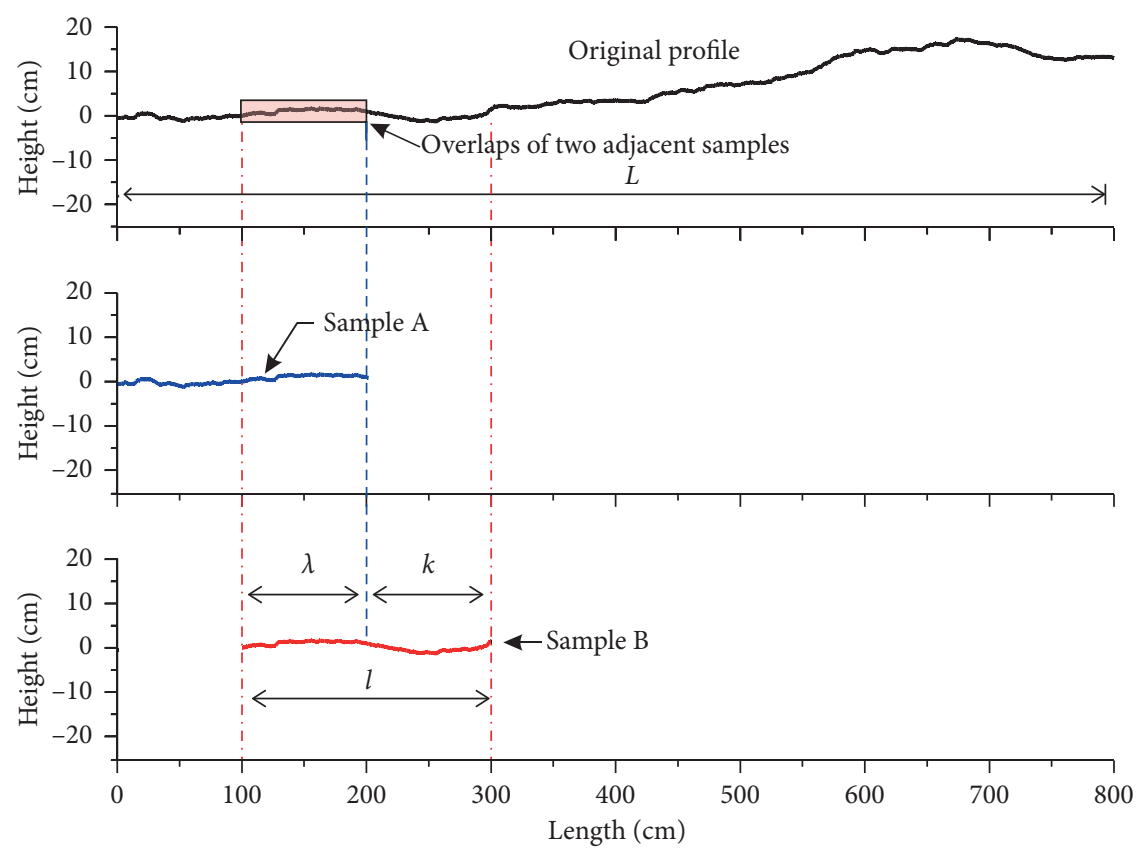

Figure 5: Schematic diagram of overlap sampling method.

3.3. Neutrosophic Function with NNs for Investigating the Scale Dependency of Joint Roughness. Smarandache [32-35] proposed $\mathrm{NN}$ under the indeterminate environments. It consisted of a certain part and an uncertain part. The former is given as $\alpha$, and the latter is expressed as $\beta I$. Then, NN can be expressed as $\mu=\alpha+\beta I$ for $\alpha, \beta \in R$. Here, $I$ denotes indeterminacy, and $R$ represents the real numbers. NN indicates a changeable interval number corresponding to different indeterminate ranges of $I \in\left[I^{-}, I^{+}\right]$. Since NN is flexible and convenient in expressing indeterminate information in indeterminate situations, it has been widely used to represent determinate and/or indeterminate information in many areas.

For any two NNs $\mu_{1}=\alpha_{1}+\beta_{1} I$ and $\mu_{2}=\alpha_{2}+\beta_{2} I$ for $\mu_{1}$, $\mu_{2} \in \mu$, and $I \in\left[\Gamma, I^{+}\right]$, we developed some basic operational laws as follows:

$$
\begin{aligned}
\mu_{1}+\mu_{2}= & \alpha_{1}+\alpha_{2}+\left(\beta_{1}+\beta_{2}\right) I=\left[\alpha_{1}+\alpha_{2}+\beta_{1} I^{-}+\beta_{2} I^{-}, \alpha_{1}+\alpha_{2}+\beta_{1} I^{+}+\beta_{2} I^{+}\right], \\
\mu_{1}+\mu_{2}= & \alpha_{1}+\alpha_{2}+\left(\beta_{1}+\beta_{2}\right) I=\left[\alpha_{1}+\alpha_{2}+\beta_{1} I^{-}+\beta_{2} I^{-}, \alpha_{1}+\alpha_{2}+\beta_{1} I^{+}+\beta_{2} I^{+}\right], \\
\mu_{1} \times \mu_{2}= & \alpha_{1} \alpha_{2}+\left(\alpha_{1} \beta_{2}+\alpha_{2} \beta_{1}\right) I+\beta_{1} \beta_{2} I^{2} \\
= & {\left[\min \left(\left(\alpha_{1}+\beta_{1} I^{-}\right)\left(\alpha_{2}+\beta_{2} I^{-}\right),\left(\alpha_{1}+\beta_{1} I^{-}\right)\left(\alpha_{2}+\beta_{2} I^{+}\right),\left(\alpha_{1}+\beta_{1} I^{+}\right)\left(\alpha_{2}+\beta_{2} I^{-}\right),\left(\alpha_{1}+\beta_{1} I^{+}\right)\left(\alpha_{2}+\beta_{2} I^{+}\right)\right),\right.} \\
& \left.\max \left(\left(\alpha_{1}+\beta_{1} I^{-}\right)\left(\alpha_{2}+\beta_{2} I^{-}\right),\left(\alpha_{1}+\beta_{1} I^{-}\right)\left(\alpha_{2}+\beta_{2} I^{+}\right),\left(\alpha_{1}+\beta_{1} I^{+}\right)\left(\alpha_{2}+\beta_{2} I^{-}\right),\left(\alpha_{1}+\beta_{1} I^{+}\right)\left(\alpha_{2}+\beta_{2} I^{+}\right)\right)\right], \\
\frac{\mu_{1}}{\mu_{2}=} & \frac{\alpha_{1}+\beta_{1} I}{\alpha_{2}+\beta_{2} I}=\frac{\left[\alpha_{1}+\beta_{1} I^{-}, \alpha_{1}+\beta_{1} I^{U}\right]}{\left[\alpha_{2}+\beta_{2} I^{-}, \alpha_{2}+\beta_{2} I^{U}\right]}=\left[\min \left(\frac{\alpha_{1}+\beta_{1} I^{-}}{\alpha_{2}+\beta_{2} I^{+}}, \frac{\alpha_{1}+\beta_{1} I^{-}}{\alpha_{2}+\beta_{2} I^{-}}, \frac{\alpha_{1}+\beta_{1} I^{+}}{\alpha_{2}+\beta_{2} I^{+}}, \frac{\alpha_{1}+\beta_{1} I^{+}}{\alpha_{2}+\beta_{2} I^{-}}\right),\right. \\
& \left.\max \left(\frac{\alpha_{1}+\beta_{1} I^{-}}{\alpha_{2}+\beta_{2} I^{+}}, \frac{\alpha_{1}+\beta_{1} I^{-}}{\alpha_{2}+\beta_{2} I^{-}}, \frac{\alpha_{1}+\beta_{1} I^{+}}{\alpha_{2}+\beta_{2} I^{+}}, \frac{\alpha_{1}+\beta_{1} I^{+}}{\alpha_{2}+\beta_{2} I^{-}}\right)\right] .
\end{aligned}
$$

According to the roughness data, it is preferred to use the mean value of all same sized joint samples to represent the joint roughness under this scale. The standard deviations have not been taken into consideration yet, which can quantify the amount of variation or dispersion of a set of joint roughness values. In Figure 1, the JRC values of the joint samples in a length of $10 \mathrm{~cm}$ are more discrete than the $20 \mathrm{~cm}$ samples. Thus, the standard value of $10 \mathrm{~cm}$ long samples should be larger than those of $20 \mathrm{~cm}$ in length. The $\mathrm{JRC}$ values ranging from $\mathrm{JRC}_{\text {mean }}-\sigma$ (lower limit) to $\mathrm{JRC}_{\text {mean }}+\sigma$ (upper limit) were chosen to describe the joint roughness properties. The range of JRC values reflected the 
majority JRC values for the samples of the same size. Generally, the upper and lower limits decreased with the length of rock joint samples increasing. Their relationships were expressed by the following logarithmic functions:

$$
\left\{\begin{array}{l}
\mathrm{JRC}_{\text {down }}=m_{1}+m_{2} \cdot \ln (l), \\
\mathrm{JRC}_{\text {up }}=m_{3}+m_{4} \cdot \ln (l),
\end{array}\right.
$$

where $\mathrm{JRC}_{\text {down }}$ and $\mathrm{JRC}_{\mathrm{up}}$ are the predicted values of both lower and upper limits, and $m_{1}, m_{2}, m_{3}$, and $m_{4}$ are the fitting coefficients.

Thus, the neutrosophic function of JRC for $I \in[0,0.5]$ is expressed as

$$
\begin{aligned}
\operatorname{JRC}(l) & =\left(m_{1}+n_{m_{1}} I\right)+\left(m_{2}+n_{m_{2}} I\right) * \ln (l) \\
& =\left(m_{1}+m_{2} \ln (l)\right)+\left(n_{m_{1}}+n_{m_{2}} \ln (l)\right) I \\
& =\left[\frac{m_{1}+m_{2} \ln (l), m_{1}+m_{2} \ln (l)+\left(n_{m_{1}}+n_{m_{2}} \ln (l)\right)}{2}\right],
\end{aligned}
$$

$$
I \in[0,0.5] .
$$

where $m_{1}$ and $m_{2}$ are the fitting coefficients of the lower bound values and $n_{m 1}$ and $n_{m 2}$ are twice the difference of the fitting coefficients between the lower and upper bound values.

According to (9), the variation trend of scale effect can be expressed by the derivative of $\operatorname{JRC}(l)$. The expression of the derivative $D_{\text {JRC }}$ is

$$
D_{\mathrm{JRC}}=\left[\frac{2 m_{2}+n_{m_{2}}}{2 l}, \frac{m_{2}}{l}\right] .
$$

\section{Application}

In this study, the natural rock joint (Figure 6) was selected from a bedding surface located in Changshan County, Zhejiang Province, China. This slate rock was slightly weathered and had a relatively smooth, planar surface. An $800 \mathrm{~cm}$ long joint profile was measured using the mechanical hand profilograph (Figure 7(a)). The obtained joint profile was digitized using the grayscale image processing technique and shown in Figure 7(b).

In order to determine a reasonable sample number, the roughness of joint profiles $10 \mathrm{~cm}, 50 \mathrm{~cm}$, and $100 \mathrm{~cm}$ long was calculated based on the relationship between the surface amplitude asperities and JRC values [35]. The approximate value of field-scale JRC is approximately determined from the measured values of amplitudes and lengths of joint profiles. The number of samples with the same size changed from 10 to 200 at an interval of 10 . The mean value and standard deviation of the samples in length of $10 \mathrm{~cm}, 50 \mathrm{~cm}$, and $100 \mathrm{~cm}$ are given in Figure 8, which shows that the statistical results (i.e., mean value and standard deviation) tend to be consistent until the sample number reaches 100 . Therefore, a sample number of 100 was taken for the joint profiles of each size in this study.

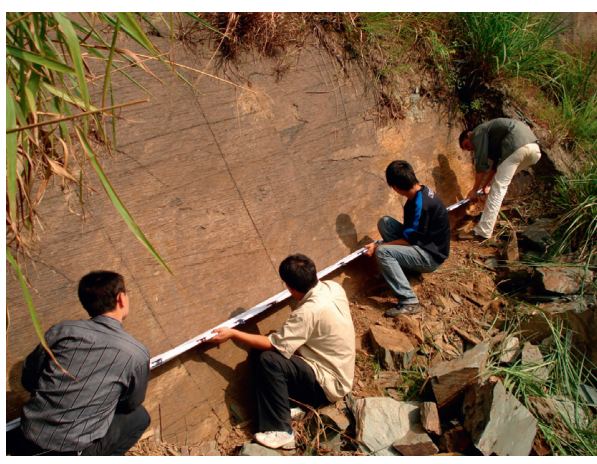

Figure 6: The slate rock joint surface.

The statistical results of the JRC values of the joint profiles with the same length were separately analyzed. The frequency distributions and cumulative probabilities of the $10 \mathrm{~cm}, 20 \mathrm{~cm}, 50 \mathrm{~cm}$, and $100 \mathrm{~cm}$ long joint samples are shown in Figure 9. They are represented graphically by characteristic bell-shaped curves. It was found that when the sample length increased, the JRC mean value and standard deviation decreased. The mean value $\mathrm{JRC}_{\text {mean }}$ and standard deviation $\sigma$ of JRC values of the $10 \mathrm{~cm}$ long samples were 10.56 and 5.34. Values ranging from $\mathrm{JRC}_{\text {mean }}-\sigma$ (lower limit) to $\mathrm{JRC}_{\text {mean }}+\sigma$ (upper limit) were chosen to describe the roughness characteristics. The lower and upper limits of $10 \mathrm{~cm}$ long samples are 5.22 and 15.91. As shown in Figure 9, $75 \%, 63 \%, 67 \%$, and $67 \%$ of the data of the samples in different lengths distributed within the interval $\left[\mathrm{JRC}_{\text {mean }}-\sigma\right.$, $\left.\mathrm{JRC}_{\text {mean }}+\sigma\right]$. The lower limits, upper limits, and JRC mean values of the joint sample in length from $10 \mathrm{~cm}$ to $300 \mathrm{~cm}$ were plotted in Figure 10. A decreasing trend was found in both the upper and lower limits as sample size progressively increased, which fit the exponential functions. Thus, $m_{1}$ and $m_{2}$ were 19.59 and -2.26 and $m_{3}$ and $m_{4}$ were 6.61 and -0.43 (Figure 10). Then,

$$
\left\{\begin{array}{l}
n_{m 1}=2 \times(19.59-6.61)=25.96 \\
n_{m 2}=2 \times(-2.26+0.43)=-3.66
\end{array}\right.
$$

Thus, the NN function JRC $(l)$ with $I \in[0,0.5]$ for describing the JRC values of different sized joint samples was

$$
\begin{aligned}
\operatorname{JRC}(l) & =(6.61+25.96 I)+(-0.43-3.66 I) * \ln (l) \\
& =[6.61-0.43 \ln (l), 19.59-2.26 \ln (l)] .
\end{aligned}
$$

Based on (12), the derivative of JRC ( $L$ ) for indicating the changing rates in different sizes was expressed as

$$
D_{\text {IRC }}=\left[\frac{-4.09}{l}, \frac{-2.26}{l}\right] .
$$

The derivative $D_{\text {JRC }}$ shows the rate of change of the JRC values relative to the change of joint profile length. $D_{\mathrm{JRC}}=0$ means that JRC is almost constant around this length. As shown in Figure 11, the derivative values increased as the sample length increased. $D_{\text {JRC }}$ of joint profile in small length changed rapidly, but it tended to be almost constant when the length of the joint profile exceeds a certain value, called the stationarity limit. It indicated that JRC values of small samples 


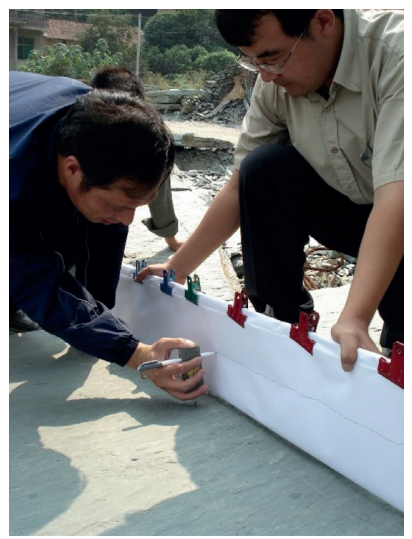

(a)

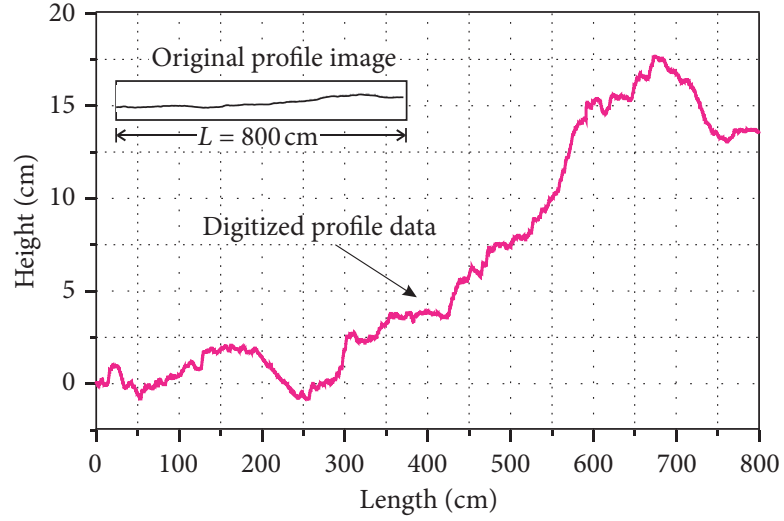

(b)

Figure 7: Measurement and digitization of large-scale rock joint roughness profiles.

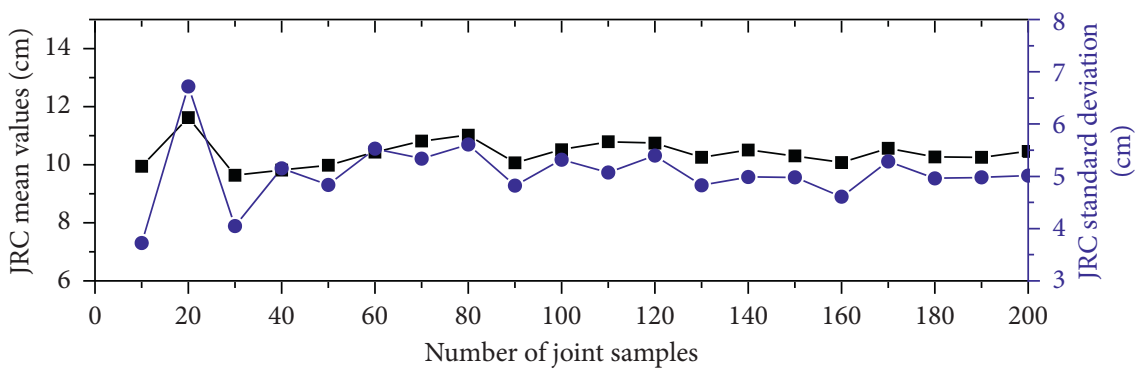

(a)

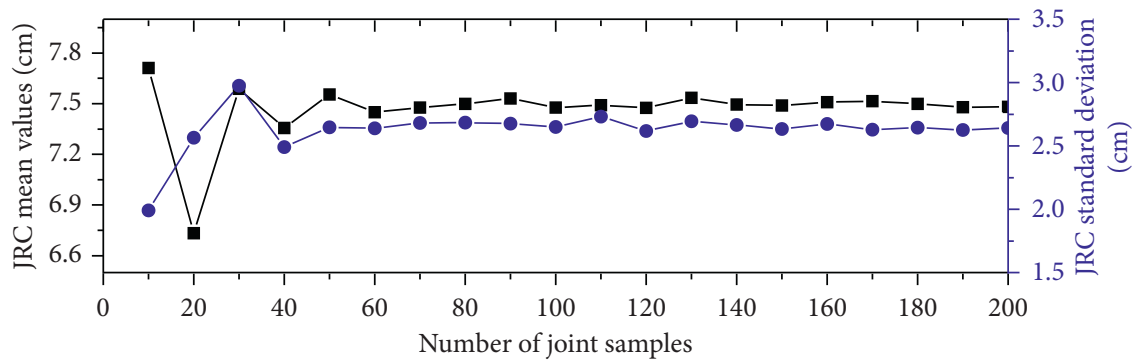

(b)

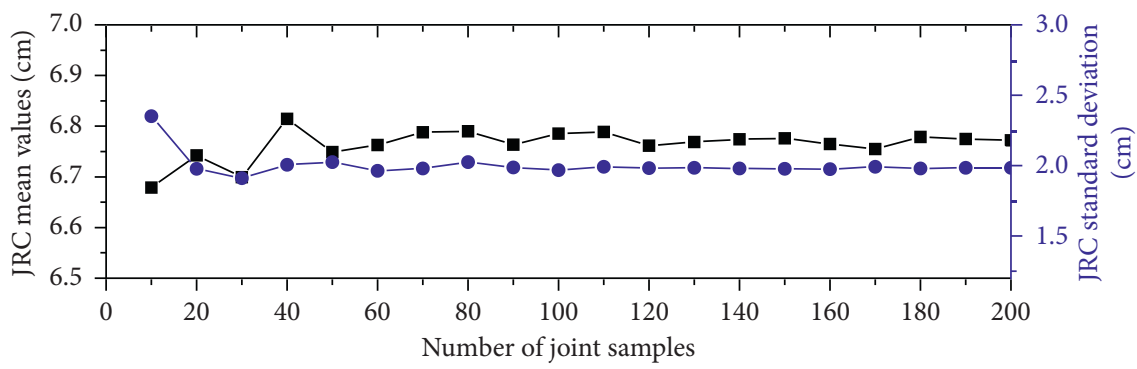

(c)

FIGURE 8: The influence of joint sample number on JRC mean values and standard deviations: (a) $10 \mathrm{~cm}$; (b) $50 \mathrm{~cm}$; (c) $100 \mathrm{~cm}$.

were more sensitive than those of larger ones. For samples smaller than this, the joint roughness was scale dependent. Beyond this limit, however, the roughness behavior was scale independent. The sample length was assumed to reach the stationarity limit once $D_{\text {IRC }}$ equal to 0.05 . Thus, here, the $80 \mathrm{~cm}$ sample length represented the stationarity limit. When the sample sizes exceeded $80 \mathrm{~cm}$, and the roughness behavior was practically scale independent. 


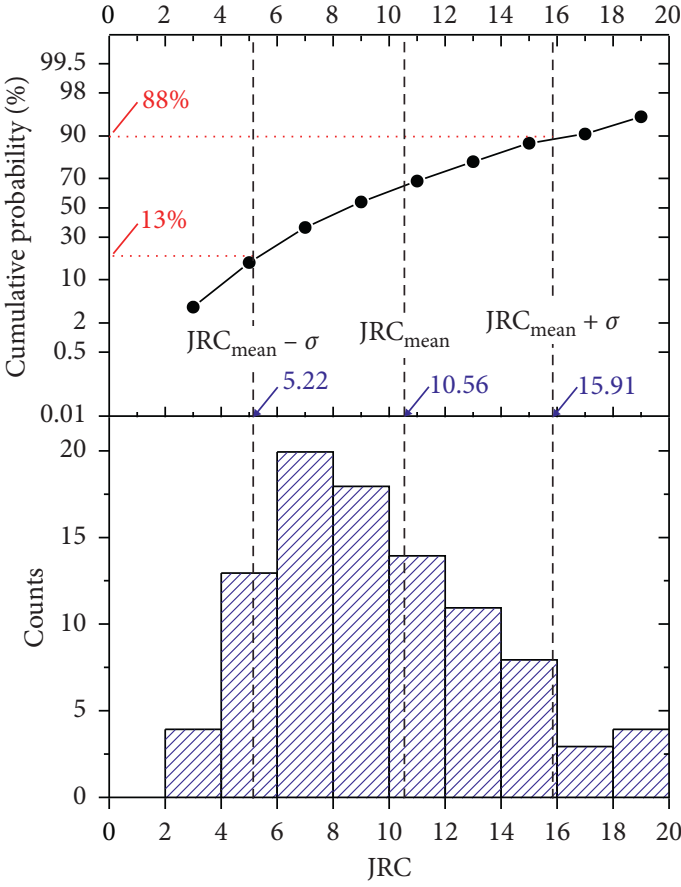

(a)

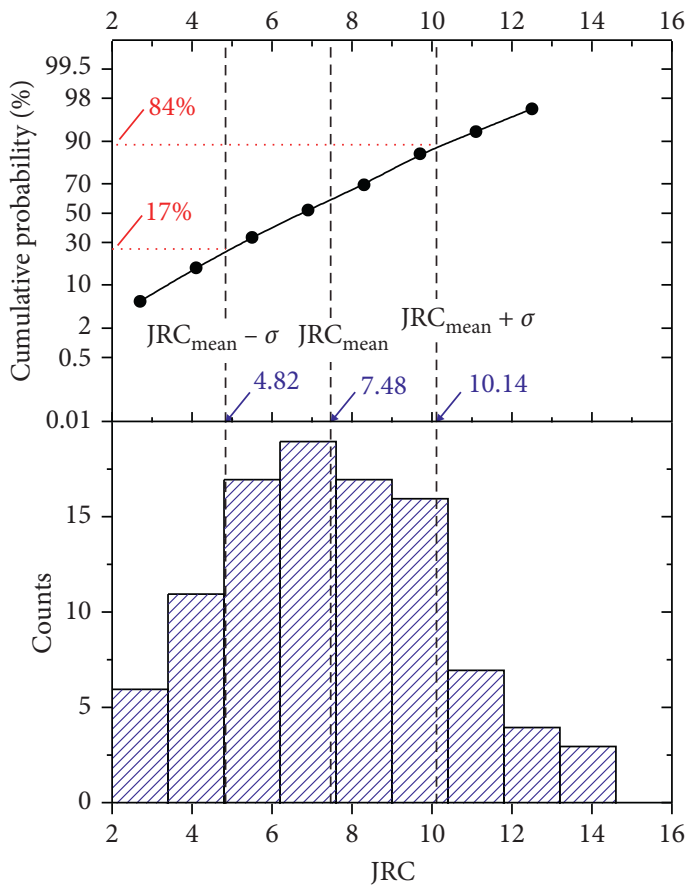

(c)

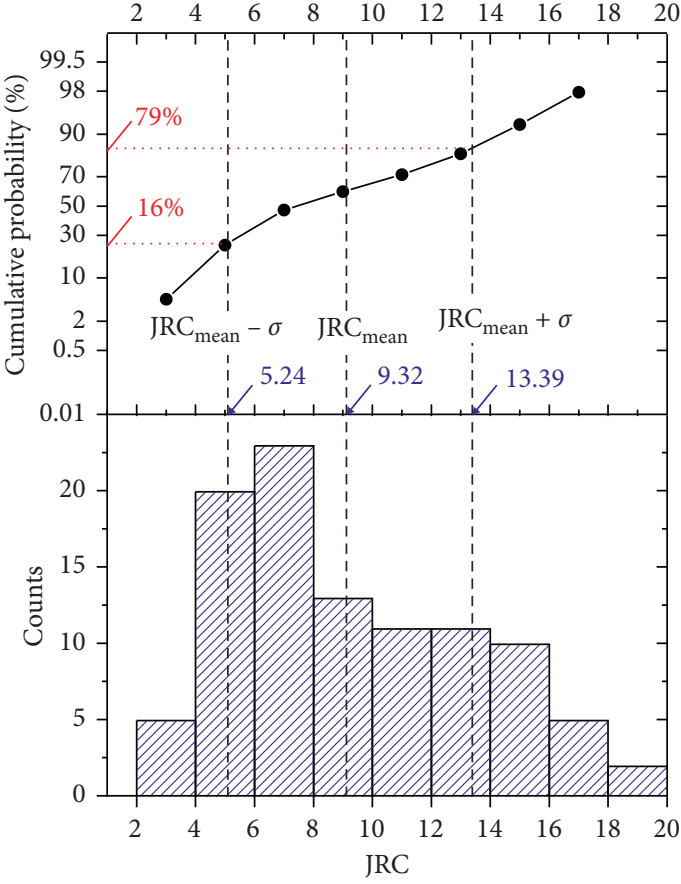

(b)

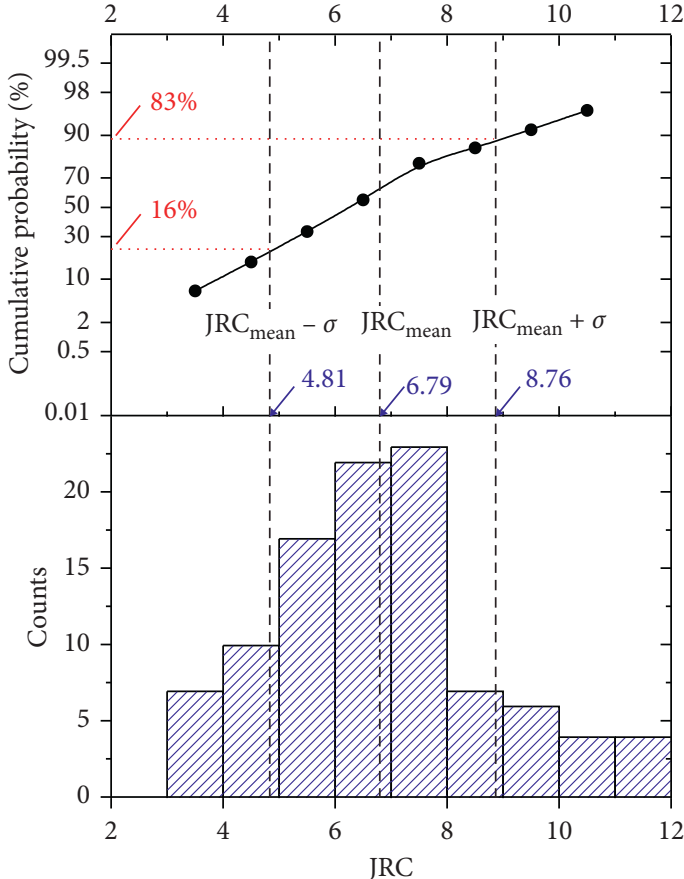

(d)

FIgURE 9: The frequency distributions and cumulative probabilities of different sized joint samples. (a) $10 \mathrm{~cm}$, (b) $20 \mathrm{~cm}$, (c) $50 \mathrm{~cm}$, and (d) $100 \mathrm{~cm}$.

\section{Conclusions and Future Work}

Improving the characterization of scale effect on rock joint roughness is an important step for predicting the mechanical behavior of the rock fractures. So far, people have paid considerable efforts to explore the joint roughness scale effect. Several limitations exist in previous studies: (1) the lack of appropriate devices for accurately and quickly measuring large joint surfaces in situ, (2) the representative of test sample was not fully considered, and (3) the statistical data of joint roughness fails to be comprehensively reflected. To address these problems, this study suggested a new method as follows.

A simple mechanical hand profilograph was adopted in this study for the measurement of large rock joint profiles. 


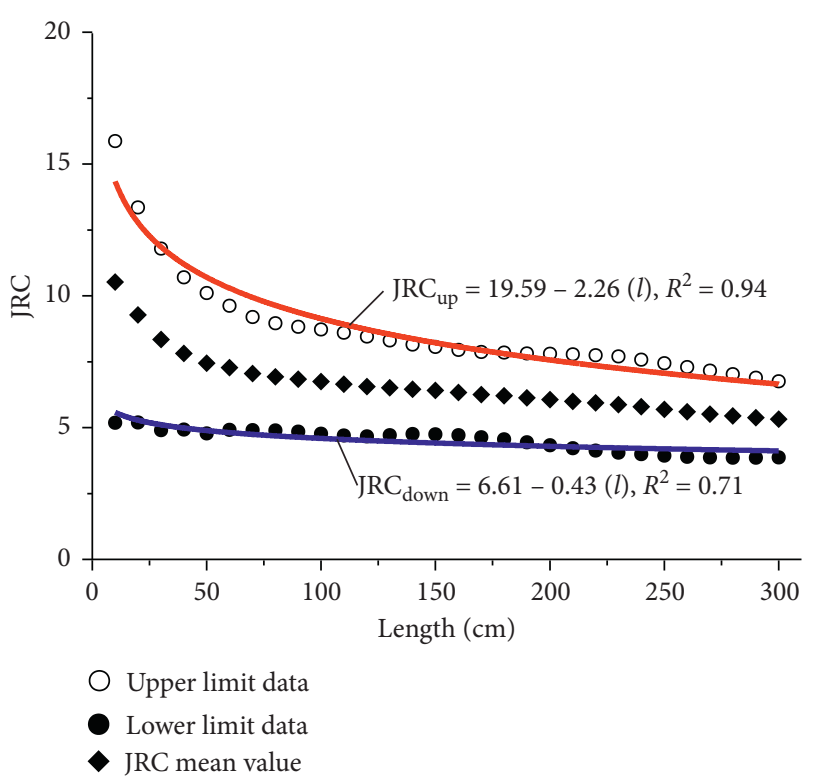

FIGURE 10: The upper and lower bounds of JRC of different sized joint samples.

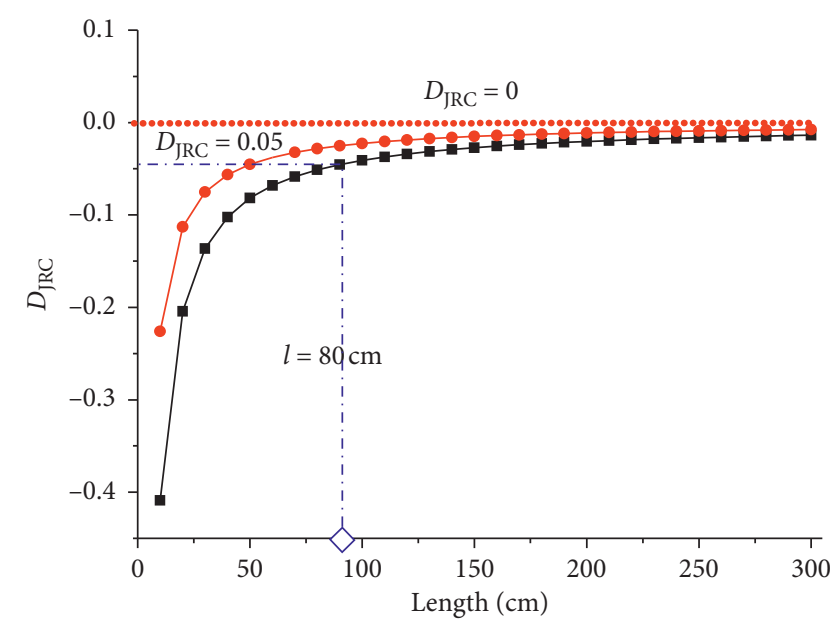

FIGURE 11: The derivative of JRC values based on neutrosophic function with NNs.

For measuring the joint profiles several meters long, a fixed board was employed, consisting of two fixed-sized, stainless steel boards that were conveniently assembled.

The roughness along different sections of a joint surface varied significantly. Consequently, a new sampling process was proposed for obtaining sufficient rock joint samples. The relationship between the number of samples and the statistical mean value and standard deviation were considered for determining a reasonable number of joint samples for statistical purposes.

The JRC statistical analysis was used to study the scale dependency of joint roughness. The neutrosophic functions $\operatorname{JRC}(l)$ were proposed for expressing the indeterminate information about the scale effect on joint roughness. The upper and lower bounds of JRC values reduced with the sample size, showing a negative scale effect on joint roughness. Based on the neutrosophic functions $\operatorname{JRC}(l)$, the variation trend of scale effect was indicated by the derivative of $\operatorname{JRC}(l)$.

The scale effect of rock joints was quantitatively expressed by neutrosophic functions, and the derivative indicated that the JRC values of small samples are more sensitive than those of large-sized examples. The stationary limit of joint roughness is $80 \mathrm{~cm}$, when the length of the samples exceeded it, and the roughness behavior was almost scale independent.

In our future work, the direct shear test will be performed for the verification of JRC estimation results of different sized joint specimens. In addition, the applications of the neutrosophic functions in other domains, such as intelligent manufacturing, machine learning, and data mining, will be conducted.

\section{Data Availability}

The data used to support the findings of this study are included within the article.

\section{Conflicts of Interest}

The authors declare that there are no conflicts of interest.

\section{Authors' Contributions}

Rui Yong proposed the main idea of this paper. Shaonan Tan and Jun Ye collected the natural rock joint samples and measured different sized joint surfaces. Shigui Du worked on joint roughness assessment and statistical analysis on the JRC values.

\section{Acknowledgments}

The study was funded by the National Natural Science Foundation of China (nos. 41502300, 41427802, and 41572299) and Zhejiang Provincial Natural Science Foundation (no. LQ16D020001).

\section{References}

[1] B. G. Chae, Y. Ichikawa, G. C. Jeong, Y. S. Seo, and B. C. Kim, "Roughness measurement of rock discontinuities using a confocal laser scanning microscope and the Fourier spectral analysis," Engineering Geology, vol. 72, no. 3-4, pp. 181-199, 2004.

[2] B. S. A. Tatone and G. Grasselli, "An investigation of discontinuity roughness scale dependency using high-resolution surface measurements," Rock Mechanics and Rock Engineering, vol. 46, no. 4, pp. 657-681, 2013.

[3] R. Yong, X. Fu, M. Huang, Q. Liang, and S.-G. Du, “A rapid field measurement method for the determination of joint roughness coefficient of large rock joint surfaces," KSCE Journal of Civil Engineering, vol. 22, no. 1, pp. 101-109, 2017.

[4] R. Yong, J. Ye, B. Li, and S.-G. Du, "Determining the maximum sampling interval in rock joint roughness measurements using Fourier series," International Journal of Rock Mechanics and Mining Sciences, vol. 101, pp. 78-88, 2018. 
[5] Y. Chen, H. Lin, X. Ding, and S. Xie, "Scale effect of shear mechanical properties of non-penetrating horizontal rocklike joints," Environmental Earth Sciences, vol. 80, no. 5, p. 192, 2021.

[6] G. Weissbach, "A new method for the determination of the roughness of rock joints in the laboratory," International Journal of Rock Mechanics and Mining Sciences \& Geomechanics Abstracts, vol. 15, no. 3, pp. 131-133, 1978.

[7] S. E. Mogilevskaya, "Methods in the study of rock jointing parameters and properties of hydraulic structure foundation," Power Technology and Engineering, vol. 38, no. 4, pp. 202-207, 2004.

[8] A. Micallef and A. T. Williams, "Shore platform denudation measurements along the Maltese coastline," Journal of Coastal Research, vol. 56, pp. 737-741, 2009.

[9] P. Alameda-Hernández, J. Jiménez-Perálvarez, J. A. Palenzuela et al., "Improvement of the JRC calculation using different parameters obtained through a new MA survey method applied to rock discontinuities," Rock Mechanics and Rock Engineering, vol. 47, no. 5, pp. 2047-2060, 2014.

[10] N. Fardin, O. Stephansson, and L. Jing, "The scale dependence of rock joint surface roughness," International Journal of Rock Mechanics and Mining Sciences, vol. 38, no. 5, pp. 659-669, 2001.

[11] Q. Feng, N. Fardin, L. Jing, and O. Stephansson, “A new method for in-situ non-contact roughness measurement of large rock fracture surfaces," Rock Mechanics and Rock Engineering, vol. 36, no. 1, pp. 3-25, 2003.

[12] W. Haneberg, "Directional roughness profiles from three dimensional photogrammetric or laser scanner point clouds," in Proceedings of the 1st Canada-US Rock Mechanics Symposium, Rock Mechanics: Meeting Society's Challenges and Demands, E. Eberhardt, D. Stead, and T. Morrison, Eds., pp. 27-31, Vancouver, Canada, May 2007.

[13] Y. Ge, P. H. S. W. Kulatilake, H. Tang, and C. Xiong, "Investigation of natural rock joint roughness," Computers and Geotechnics, vol. 55, pp. 290-305, 2014.

[14] K. Khoshelham, D. Altundag, D. Ngan-Tillard, and M. Menenti, "Influence of range measurement noise on roughness characterization of rock surfaces using terrestrial laser scanning," International Journal of Rock Mechanics and Mining Sciences, vol. 48, no. 8, pp. 1215-1223, 2011.

[15] S. G. Du, "Research on complexity of surface undulating shapes of rock joints," Journal of China University of Geosciences, vol. 9, no. 1, pp. 88-91, 1998.

[16] J. Ye, R. Yong, Q.-F. Liang, M. Huang, and S.-G. Du, "Neutrosophic functions of the joint roughness coefficient and the shear strength: a case study from the pyroclastic rock mass in Shaoxing City, China," Mathematical Problems in Engineering, vol. 2016, Article ID 4825709, 9 pages, 2016.

[17] X. G. Liu, W. C. Zhu, Q. L. Yu, S. J. Chen, and R. F. Li, "Estimation of the joint roughness coefficient of rock joints by consideration of two-order asperity and its application in double-joint shear tests," Engineering Geology, vol. 220, pp. 243-255, 2017.

[18] N. Barton, "Scale effects or sampling bias?" in Proceedings of the ISRM Workshop on Scale Effects in Rock Mass, pp. 31-55, Loen, Norway, 1991.

[19] S. Du, Y. Hu, and X. Hu, "Measurement of joint roughness coefficient by using profilograph and roughness ruler," Journal of Earth Science, vol. 20, no. 5, pp. 890-896, 2009.

[20] S. M. Hsiung, A. Ghosh, M. P. Ahola, and A. H. Chowdhury, "Assessment of conventional methodologies for joint roughness coefficient determination," International Journal of
Rock Mechanics and Mining Sciences \& Geomechanics Abstracts, vol. 30, no. 7, pp. 825-829, 1993.

[21] S. J. Chen, W. C. Zhu, S. X. Liu, F. Zhang, and L. F. Guo, "Anisotropy and size effects of surface roughness of rock joints," Chinese Journal of Rock Mechanics and Engineering, vol. 34, no. 1, pp. 57-65, 2015, in Chinese.

[22] L. Wang, C. Wang, S. Khoshnevisan, Y. Ge, and Z. Sun, "Determination of two-dimensional joint roughness coefficient using support vector regression and factor analysis," Engineering Geology, vol. 231, pp. 238-251, 2017.

[23] Y. Li, S. Sun, and H. Yang, "Scale dependence of waviness and unevenness of natural rock joints through fractal analysis," Geofluids, vol. 2020, Article ID 8818815, 18 pages, 2020.

[24] F. Yan, L. Ban, C. Qi, R. Shan, and H. Yuan, "Research on the anisotropy, size effect, and sampling interval effect of joint surface roughness," Arabian Journal of Geosciences, vol. 13, pp. 1-9, 2020.

[25] ISRM "International, "International society for rock mechanics commission on standardization of laboratory and field tests," International Journal of Rock Mechanics and Mining Sciences \& Geomechanics Abstracts, vol. 15, no. 6, pp. 319-368, 1978.

[26] B. Rousseau, P. Rivard, A. Marache, G. Ballivy, and J. Riss, "Limitations of laser profilometry in measuring surface topography of polycrystalline rocks," International Journal of Rock Mechanics and Mining Sciences, vol. 52, pp. 56-60, 2012.

[27] J. Mah, C. Samson, S. D. McKinnon, and D. Thibodeau, "3D laser imaging for surface roughness analysis," International Journal of Rock Mechanics and Mining Sciences, vol. 58, pp. 111-117, 2013.

[28] G. L. Morelli, "On joint roughness: measurements and use in rock mass characterization," Geotechnical and Geological Engineering, vol. 32, no. 2, pp. 345-362, 2014.

[29] R. Yong, J. Qin, M. Huang, S. Du et al., "An innovative sampling method for determining the scale effect of rock joints," Rock Mechanics and Rock Engineering, vol. 52, pp. 1-12, 2018.

[30] G. Zhang, M. Karakus, H. Tang, Y. Ge, and L. Zhang, "A new method estimating the 2D Joint Roughness Coefficient for discontinuity surfaces in rock masses," International Journal of Rock Mechanics and Mining Sciences, vol. 72, pp. 191-198, 2014.

[31] R. Yong, L. Gu, J. Ye et al., "Neutrosophic function with NNs for analyzing and expressing anisotropy characteristic and scale effect of joint surface roughness," Mathematical Problems in Engineering, vol. 2019, pp. 1-11, 2019.

[32] F. Smarandache, Neutrosophy: Neutrosophic Probability, Set, and Logic, pp. 56-72, American Research Press, Santa Fe, NM, USA, 1998.

[33] F. Smarandache, Introduction to Neutrosophic Measure, Neutrosophic Integral, and Neutrosophic Probability, Sitech \& Education Publisher, Columbus, OH, USA, 2013.

[34] F. Smarandache, Introduction to Neutrosophic Statistics, Sitech \& Education Publisher, Columbus, OH, USA, 2014.

[35] N. Barton and S. Bandis, "Effects of soluble salts on the threshold shear velocity of fine sand," International Journal of Rock Mechanics and Mining Sciences \& Geomechanics Abstracts, vol. 19, no. 2, 1982. 